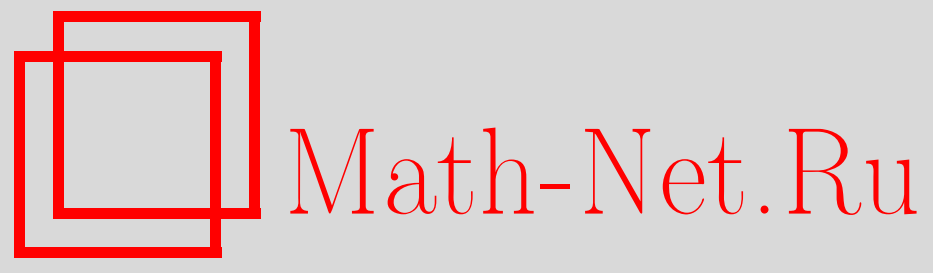

М. Ю. Ливщиц, А. П. Сизиков, Об одном методе поиска глобального экстремума непрерывной функции на симплексе, Вестн. Сам. гос. техн. унта. Сер. Физ.-мат. науки, 2016, номер 4, 755-768

DOI: https://doi.org/10.14498/vsgtu1500

Использование Общероссийского математического портала MathNet.Ru подразумевает, что вы прочитали и согласны с пользовательским соглашением http://www . mathnet.ru/rus/agreement

Параметры загрузки:

IP : 54.224 .60 .19

26 апреля 2023 г., 09:48:27

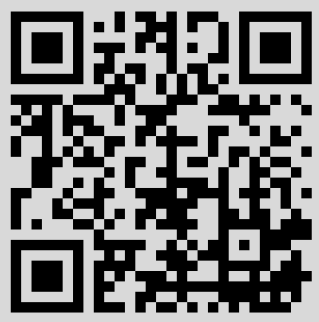


УДК 519.853.4

\title{
ОБ ОДНОМ МЕТОДЕ ПОИСКА ГЛОБАЛЬНОГО ЭКСТРЕМУМА НЕПРЕРЫВНОЙ ФУНКЦИИ НА СИМПЛЕКСЕ
}

\author{
М. Ю. Лившич, А. П. Сизиков \\ Самарский государственный технический университет, \\ Россия, 443100, Самара, ул. Молодогвардейская, 244.
}

\begin{abstract}
Аннотация
Рассматривается невыпуклая задача математического программирования, допустимой областью которой является симплекс. Для приближенного решения задачи предложен двухэтапный алгоритм. На первом этапе определяется область глобального оптимума, на втором осуществляется локальная «доводка» решения. Первый этап реализуется методом $\Psi$-преобразования, который менее чувствителен к размерности задачи, чем прямой случайный поиск. Метод состоит в построении и использовании $\Psi$-функции. Построение $\Psi$-функции осуществляется эмпирически, по результатам статистических испытаний. Для проведения испытаний используется генератор случайных точек, равномерно распределенных в допустимой области. Получение равномерного распределения в симплексе осуществляется путем аффинных и линейных преобразований точек, равномерно распределенных в единичном гиперкубе. Для уточнения приближенного решения, полученного на первом этапе, применяется метод отражения правильных симплексов. Алгоритм представляет собой череду серий. Каждая очередная серия начинается с уменьшения длины ребра «рабочего» симплекса и переноса последнего в область лучшей точки предыдущей серии. Рассматриваемая задача имеет важное прикладное значение. $\mathrm{K}$ ней сводятся задачи смешения, возникающие в нефтепереработке, нефтехимии, строительстве. В статье представлен пример использования разработанного алгоритма для оптимизации компонентного состава углеводородной смеси.
\end{abstract}

Ключевые слова: оптимизация, невыпуклые задачи, метод $\Psi$-преобразования, равномерное распределение в симплексе, многокомпонентные смеси.

(C) 2016 Самарский государственный технический университет.

\section{Образец для цитирования}

Л и в ш и ц М. Ю., С и з и к о в А. П. Об одном методе поиска глобального экстремума непрерывной функции на симплексе // Вестн. Сам. гос. техн. ун-та. Сер. Физ.-мат. науки, 2016. T. 20, № 4. C. 755-768. doi: 10.14498/vsgtu1500.

\section{Сведения об авторах}

Михаил Юръевич Лившии, (д.т.н., проф.; mikhaillivshits@gmail.com), заведующий кафедрой, каф. управления и системного анализа теплоэнергетических и социотехнических комплексов.

Александр Павлович Сизиков (к.э.н., доц.; apsizikov@mail.ru; автор, ведущий переписку), докторант, каф. управления и системного анализа теплоэнергетических и социотехнических комплексов. 
В широкой области технических приложений возникает необходимость решения нелинейной и, в общем случае, невыпуклой задачи:

$$
\begin{gathered}
f(x) \rightarrow \max , \\
\left\{\begin{array}{l}
\sum_{j=1}^{m} x_{j}=1 \\
x_{j} \geqslant 0, j=1,2, \ldots, m,
\end{array}\right.
\end{gathered}
$$

где $f(x)$ - некоторая $m$-мерная непрерывная функция $x=\left(x_{1}, x_{2}, \ldots, x_{m}\right)$.

Для решения невыпуклых оптимизационных задач наиболее часто используют стохастический подход. Он включает широкий спектр методов от простого случайного поиска до различных рандомизированных эвристик [1-3]. Все эти методы так или иначе используют глобальный и локальный поиск в различных реализациях и сочетаниях.

В методе имитации отжига переход от глобального к локальному поиску осуществляется постепенно [4,5]. На начальном этапе осуществляется случайный поиск по всей допустимой области. Но затем, оставаясь случайным, он все более и более локализуется, становится избирательным. В методе кроссэнтропии [6-8] идея постепенной локализации поиска реализуется через механизм изменения распределения поисковой выборки с тем, чтобы перспективные варианты выпадали с большей вероятностью. Существуют методы, использующие так называемый «мультистарт» локальных поисков (детерминированных или рандомизированных) сразу из множества точек области определения $[9,10]$. Для решения некоторых трудно формализуемых задач эффективны генетические алгоритмы [11-13]. В целом же эти алгоритмы обладают всеми достоинствами и недостатками рандомизированных эвристик [14].

Авторы предлагают для решения задачи (1), (2) модификацию известного метода $\Psi$-преобразования [15-17], сочетающего детерминированный и стохастический подходы. По сравнению с методами [1-13] предложенный метод менее чувствителен к размерности задачи, поскольку не предполагает прямого поиска.

Для задачи

$$
\max \{f(x) \mid x \in E\}
$$

где $E$ - некоторое подмножество евклидова пространства, в рассмотрение вводится функция $\Psi(\zeta)$, которая определяется как мера объема множества

$$
\tilde{E}(\zeta)=\{x \mid x \in E, f(x) \geqslant \zeta\} .
$$

Поскольку $\Psi(\zeta)$ является монотонно убывающей, ноль этой функции соответствует решению задачи. Определение функции $\Psi(\zeta)$ в аналитическом виде затруднительно, поэтому некоторое ее приближение строится по результатам статистических испытаний. О значении $\Psi(\zeta)$ можно судить по относительному количеству случайных точек, попадающих в $\tilde{E}(\zeta)$, при условии, что точки равномерно распределены во множестве $E$. Поэтому в поставленной задаче (1), (2), прежде чем использовать алгоритм $\Psi$-преобразования, необходимо при статистических испытаниях решить вопрос генерирования случайных точек, равномерно распределенных в области (2).

Правильный $(m-1)$-мерный симплекс $(2)$ можно поместить в соответствующий $(m-1)$-мерный единичный гиперкуб в $\mathbb{R}^{m-1}$, получение равномерного 
распределения случайных чисел в котором - задача тривиальная. Поскольку симплекс является подмножеством гиперкуба, точки, попадающие в симплекс, также равномерно распределены. Однако для поставленной задачи $(1),(2)$ этот путь непродуктивен. Как известно, объем правильного $(m-1)$ мерного симплекса со стороной единичной длины равен

$$
\frac{1}{(m-1) !} \sqrt{\frac{m}{2^{m-1}}},
$$

и при росте $m$ быстро стремится к нулю. Соответственно, стремится к нулю и вероятность того, что случайная точка, сгенерированная в гиперкубе, окажется в симплексе. Поэтому для получения случайных допустимых вариантов используем алгоритм [18,19], который обеспечивает перенос равномерно распределенных случайных точек в симплекс (2) путем аффинных и линейных преобразований, не влияющих на закон распределения.

\section{Алгоритм 1.}

1. Генерируются $(m-1)$ случайных чисел, равномерно распределенных на единичном отрезке. Эти числа нумеруются в порядке возрастания:

$$
\xi_{1} \leqslant \xi_{2} \leqslant \ldots \leqslant \xi_{m-1}
$$

2. Формируется вектор

$$
\xi^{\prime}=\left(\xi_{1}^{\prime}, \xi_{2}^{\prime}, \ldots, \xi_{m-1}^{\prime}\right),
$$

где $\xi_{1}^{\prime}=\xi_{1}, \xi_{2}^{\prime}=\xi_{2}-\xi_{1}, \ldots, \xi_{m-1}^{\prime}=\xi_{m-1}-\xi_{m-2}$. Этот вектор, как показано в работе [18], равномерно распределен на множестве

$$
M=\left\{x \in \mathbb{R}^{m-1} \mid x_{1} \geqslant 0, x_{2} \geqslant 0, \ldots, x_{m-1} \geqslant 0, \sum_{i=1}^{m-1} x_{i} \leqslant 1\right\} .
$$

3. Все угловые точки многогранника $M$, кроме нулевой, лежат на расстоянии $\sqrt{2}$ друг от друга. Многогранник $M$ растягивается до правильного $\tilde{M}$. Для этого перенесем вершину, лежащую в начале координат, так, чтобы расстояние от нее до всех остальных вершин составляло также $\sqrt{2}$. Это будет точка с координатами $\left(x_{1}^{0}, x_{2}^{0}, \ldots, x_{m-1}^{0}\right)$, где $x_{i}^{0}=$ $=r=(1-\sqrt{m}) /(m-1), i=1,2, \ldots, m-1$. Получился многогранник, конгруэнтный $(m-1)$-мерному симплексу в $m$-мерном пространстве. Обозначим через $\underset{\tilde{\xi}}{\tilde{\xi}}$ точку множества $\tilde{M}$, отображающую точку $\xi^{\prime}$ множества $M$. Точку $\tilde{\xi}$ определяем в результате отражения $\xi^{\prime}$ относительно гиперплоскости

$$
\left\{x \in \mathbb{R}^{m-1} \mid \sum_{i=1}^{m-1} x_{i}=1\right\} .
$$

Для этого сначала находим проекцию $\xi^{\prime}$ на эту гиперплоскость:

$$
\breve{\xi}_{i}=\xi_{i}^{\prime}+\Delta, \quad i=1,2, \ldots, m-1,
$$

где $\Delta$ определяем из условия

$$
\sum_{i=1}^{m-1} \breve{\xi}_{i}=1
$$


по формуле

$$
\Delta=\frac{1}{m-1}\left(1-\sum_{i=1}^{m-1} \xi_{i}^{\prime}\right)
$$

Отражение находим с учетом того, что коэффициент растяжения равен $\sqrt{m}$ :

$$
\tilde{\xi}=\breve{\xi}+\sqrt{m}\left(\xi^{\prime}-\breve{\xi}\right)
$$

Это аффинное преобразование не оказывает влияния на закон распределения, поэтому точка $\tilde{\xi}$ имеет равномерное распределение в $\tilde{M}$.

4. Точка $\tilde{\xi}$ переносится в $(m-1)$-мерный симплекс $m$-мерного пространства переменных задачи (1), (2). Для этого представляем ее как выпуклую линейную комбинацию угловых точек $\tilde{M} m$-мерного пространства. Коэффициенты этой комбинации $\alpha_{1}, \alpha_{2}, \ldots, \alpha_{m}$ есть не что иное, как новые координаты точки $\alpha$. Они определяются следующим образом:

$$
\left(\begin{array}{c}
\alpha_{1} \\
\alpha_{2} \\
\cdots \\
\alpha_{m-1} \\
\alpha_{m}
\end{array}\right)=\left(\begin{array}{ccccc}
1 & 0 & \ldots & 0 & r \\
0 & 1 & \ldots & 0 & r \\
\ldots & \ldots & \ldots & \ldots & \ldots \\
0 & 0 & \ldots & 1 & r \\
1 & 1 & \ldots & 1 & 1
\end{array}\right)^{-1}\left(\begin{array}{c}
\tilde{\xi}_{1} \\
\tilde{\xi}_{2} \\
\ldots \\
\tilde{\xi}_{m-1} \\
1
\end{array}\right)
$$

Теперь вернемся к методу $\Psi$-преобразования.

\section{Алгоритм 2 .}

1. Выполняется серия случайных проб, число которых $N$ должно быть достаточным для получения надежных статистических оценок. На каждой пробе $k=1,2, \ldots, N$ с помощью алгоритма 1 генерируется допустимое значение аргумента $x^{(k)}$ и находится $f\left(x^{(k)}\right)$. По результатам всех проб определяются среднее арифметическое $f_{c}$ и максимальное $f_{m}$ наблюдаемых значений целевой функции:

$$
f_{c}=\frac{1}{N} \sum_{k=1}^{N} f\left(x^{(k)}\right), \quad f_{m}=\max \left\{f\left(x^{(1)}\right), f\left(x^{(2)}\right), \ldots, f\left(x^{(N)}\right)\right\} .
$$

Отрезок $\left[f_{c}, f_{m}\right]$ разбивается на $L$ (порядка десяти) уровней

$$
\zeta=\left(\zeta_{1}, \zeta_{2}, \ldots, \zeta_{L}\right)
$$

где

$$
\zeta_{l}=f_{c}+(l-1)\left(f_{m}-f_{c}\right) L^{-1}
$$

2. Выполняется вторая серия случайных проб. Для каждого уровня $l=$ $=1,2, \ldots, L$ подсчитывается $n_{l}$ - число проб, в которых выполнилось условие

$$
f\left(x^{(k)}\right) \geqslant \zeta_{l}
$$

и средняя координата:

$$
\bar{x}_{i l}=\frac{1}{n_{l}} \sum_{k=1}^{N} \delta_{l} x_{i}^{(k)}, \quad i=1,2, \ldots, m, \quad \delta_{l}=\left\{\begin{array}{l}
1, f\left(x^{(k)}\right) \geqslant \zeta_{l}, \\
0, f\left(x^{(k)}\right)<\zeta_{l} .
\end{array}\right.
$$


По результатам формируются последовательности:

$$
\begin{gathered}
\psi=\left\{\psi_{l}\right\}_{l=1}^{L}, \quad \text { где } \quad \psi_{l}=n_{l} N^{-1}, \\
\tilde{x}_{i}=\left\{\bar{x}_{i l}\right\}_{l=1}^{L}, \quad i=1,2, \ldots, m .
\end{gathered}
$$

3. Определяются квадратичные тренды. Пусть

$$
\Lambda=\left(\begin{array}{ccc}
1 & 1 & 1 \\
1 & 2 & 4 \\
\ldots & \ldots & \ldots \\
1 & L & L^{2}
\end{array}\right)
$$

тогда параметры $a=\left(a_{0}, a_{1}, a_{2}\right)^{\top}$ тренда $\psi(l)=a_{0}+a_{1} l+a_{2} l^{2}$ рассчитываются по формуле

$$
a=\left(\Lambda^{\top} \Lambda\right)^{-1} \Lambda^{\top} \psi^{\top}
$$

Аналогично рассчитываются параметры $b_{i}=\left(b_{i 0}, b_{i 1}, b_{i 2}\right)^{\top}$ тренда $\tilde{x}_{i}(l)=$ $=b_{i 0}+b_{i 1} l+b_{i 2} l^{2}$ :

$$
b_{i}=\left(\Lambda^{\top} \Lambda\right)^{-1} \Lambda^{\top} \tilde{x}_{i}^{\top} .
$$

4. Определяется наименьший положительный корень $l^{*}$ уравнения

$$
\psi(l)=0 .
$$

Его существование обусловлено тем, что $\psi(l)$ на отрезке $[1, L]$ представляет собой тренд последовательности (3), монотонно убывающей до значения, близкого к нулю. Подстановкой $l^{*}$ в тренды $\tilde{x}(l)$ находится приближенное решение задачи:

$$
x_{i}^{*}=\tilde{x}_{i}\left(l^{*}\right), \quad i=1,2, \ldots, m .
$$

Назначение описанного алгоритма 2 - войти в область глобального экстремума. Предположим, что это удалось. Перейдем к локальному поиску самого экстремума. Методы, предполагающие использование производных первого или второго порядков (метод сопряженных градиентов, метод Ньютона), здесь неприменимы по причине возможной негладкости целевой функции $f(x)$. В этом случае необходимо использовать метод, для реализации которого достаточно значений самой целевой функции $f(x)$. Например, последовательное отражение симплексов [20].

Классический метод последовательного отражения симплексов - метод поиска экстремума в $m$-мерном евклидовом пространстве с помощью $m$-мерных симплексов [20]. В задаче $(1),(2)$ речь идет о поиске экстремума в $(m-1)$ мерном симплексе с помощью $(m-1)$-мерных же симплексов.

Построим в $m$-мерном евклидовом пространстве правильный $m$-мерный симплекс, одна из вершин которого (назовем ее нулевой) лежит в начале координат. Пусть длина ребра равна $\Delta x$, тогда координаты вершин соответствуют столбцам матрицы:

$$
\left(\begin{array}{ccccc}
x_{1}^{(0)} & x_{1}^{(1)} & x_{1}^{(2)} & \ldots & x_{1}^{(m)} \\
x_{2}^{(0)} & x_{2}^{(1)} & x_{2}^{(2)} & \ldots & x_{2}^{(m)} \\
\ldots & \ldots & \ldots & \ldots & \ldots \\
x_{m}^{(0)} & x_{m}^{(1)} & x_{m}^{(2)} & \ldots & x_{m}^{(m)}
\end{array}\right)=\left(\begin{array}{ccccc}
0 & \widehat{q} & q & \ldots & q \\
0 & q & \widehat{q} & \ldots & q \\
\ldots & \ldots & \ldots & \ldots & \ldots \\
0 & q & q & \ldots & \bar{q}
\end{array}\right)
$$


где

$$
\widehat{q}=\frac{\Delta x}{m \sqrt{2}}(\sqrt{m+1}+m-1), \quad q=\frac{\Delta x}{m \sqrt{2}}(\sqrt{m+1}-1) .
$$

Перенесем этот симплекс из начала координат так, чтобы все его вершины, кроме $x^{(0)}$, расположились на гиперплоскости

$$
x_{1}+x_{2}+\cdots+x_{m}=1
$$

и чтобы $(m-1)$-мерный симплекс с вершинами $x^{(1)}, x^{(2)}, \ldots, x^{(m)}$ оказался в области начала поиска.

При параллельном переносе нулевой вершины в точку $\tilde{x}^{(0)}$ координаты остальных вершин становятся следующими:

$$
\tilde{x}^{(k)}=\tilde{x}^{(0)}+x^{(k)}, \quad k=1,2, \ldots, m .
$$

Найдем $\tilde{x}^{(0)}$ из условия

$$
\tilde{x}_{1}^{(k)}+\tilde{x}_{2}^{(k)}+\cdots+\tilde{x}_{m}^{(k)}=1 .
$$

Достаточно взять какую-нибудь одну вершину, например, первую:

$$
\begin{aligned}
\sum_{i=1}^{m} \tilde{x}_{i}^{(1)} & =\sum_{i=1}^{m} \tilde{x}_{i}^{(0)}+\frac{\Delta x}{m \sqrt{2}}(\sqrt{m+1}+m-1)+\sum_{i=2}^{m} \frac{\Delta x}{m \sqrt{2}}(\sqrt{m+1}-1)= \\
= & \sum_{i=1}^{m} \tilde{x}_{i}^{(0)}+\frac{\Delta x}{m \sqrt{2}}\left(\sqrt{m+1}+m-1+\sum_{i=1}^{m-1}(\sqrt{m+1}-1)\right)= \\
& =\sum_{i=1}^{m} \tilde{x}_{i}^{(0)}+\frac{\Delta x}{m \sqrt{2}} m \sqrt{m+1}=1 .
\end{aligned}
$$

Отсюда получаем

$$
\sum_{i=1}^{m} \tilde{x}_{i}^{(0)}=1-\frac{\Delta x}{\sqrt{2}} \sqrt{m+1}
$$

Определим начальное расположение $m$-мерного симплекса, привязав его к точке $x^{*}$, полученной методом $\Psi$-преобразования. В этом случае координаты нулевой вершины симплекса определятся следующим образом:

$$
\tilde{x}_{i}^{(0)}=x_{i}^{*}\left(1-\frac{\Delta x}{\sqrt{2}} \sqrt{m+1}\right), \quad i=1,2, \ldots, m .
$$

Координаты остальных вершин, которые и образуют начальный «рабочий» симплекс, представлены столбцами матрицы:

$$
\left(\begin{array}{cccc}
\tilde{x}_{1}^{(1)} & \tilde{x}_{1}^{(2)} & \ldots & \tilde{x}_{1}^{(m)} \\
\tilde{x}_{2}^{(1)} & \tilde{x}_{2}^{(2)} & \ldots & \tilde{x}_{2}^{(m)} \\
\ldots & \ldots & \ldots & \ldots \\
\tilde{x}_{m}^{(1)} & \tilde{x}_{m}^{(2)} & \ldots & \tilde{x}_{m}^{(m)}
\end{array}\right)
$$


где

$$
\tilde{x}_{i}^{(k)}=x_{i}^{*}\left(1-\frac{\Delta x}{\sqrt{2}} \sqrt{m+1}\right)+\left\{\begin{array}{l}
\frac{\Delta x}{m \sqrt{2}}(\sqrt{m+1}+m-1), \quad i=k, \\
\frac{\Delta x}{m \sqrt{2}}(\sqrt{m+1}-1), \quad i \neq k
\end{array}\right.
$$

Процесс поиска состоит в реализации последовательности шагов, на каждом из которых происходит построение нового «рабочего» симплекса путем отражения вершины с минимальным значением целевой функции $f(x)$ от противоположной грани исходного симплекса. Пусть $v \in[1, m]-$ номер такой вершины, тогда отраженная вершина определяется по формуле

$$
\breve{x}^{(v)}=\frac{2}{m-1}\left(\sum_{k=1}^{m} x^{(k)}-x^{(v)}\right)-x^{(v)} .
$$

Покажем, что $\breve{x}^{(v)}$ лежит на гиперплоскости $x_{1}+x_{2}+\cdots+x_{m}=1$. Действительно,

$$
\begin{aligned}
\sum_{i=1}^{m} \breve{x}_{i}^{(v)} & =\frac{2}{m-1} \sum_{i=1}^{m}\left(\sum_{k=1}^{m} x_{i}^{(k)}-x_{i}^{(v)}\right)-\sum_{i=1}^{m} x_{i=1}^{(v)}= \\
= & \frac{2}{m-1}\left(\sum_{k=1}^{m} \sum_{i=1}^{m} x_{i}^{(k)}-\sum_{i=1}^{m} x_{i}^{(v)}\right)-\sum_{i=1}^{m} x_{i}^{(v)}=\frac{2}{m-1}(m-1)-1=1 .
\end{aligned}
$$

Поскольку поиск проводится в ограниченном пространстве, может оказаться, что $\breve{x}^{(v)}$ выходит за допустимую область. Предположим для простоты, что на текущем шаге алгоритма выполняется

$$
f\left(x^{(1)}\right)<f\left(x^{(2)}\right)<\cdots<f\left(x^{(m)}\right)
$$

Тогда нужно последовательно перебирать вершины до тех пор, пока не получим допустимого отражения. Отражение лучшей точки будет признаком окончания текущей серии. Следующая серия начинается с уменьшения длины ребра $\Delta x$ и переноса нового «рабочего» симплекса в область лучшей точки предыдущей серии.

Описанная методика может быть использована для поиска оптимального компонентного состава различных смесей, в частности, для расчета рецептур смешения товарных нефтепродуктов. Некоторые показатели качества смесей рассчитываются по специальным, иногда довольно сложным, эмпирическим формулам [21,22].

Пусть $Q$ - множество индексов контролируемых показателей некоторой смеси, и пусть $\phi_{q}(x)$ - зависимость (линейная или нелинейная) $q$-того показателя смеси от компонентов смешения, взятых в пропорциях $x=\left(x_{1}, x_{2}, \ldots, x_{m}\right)$. Тогда задачу смешения можно сформулировать так:

$$
C=\sum_{j=1}^{m} c_{j} x_{j} \rightarrow \min
$$




$$
\left\{\begin{array}{l}
\phi_{q}(x) \in\left[p_{q}^{-}, p_{q}^{+}\right], \quad q \in Q \\
\sum_{j=1}^{m} x_{j}=1 \\
x_{j} \geqslant 0, \quad j=1,2, \ldots, m
\end{array}\right.
$$

где $c_{j}$ - цена $j$-того компонента; $p_{q}^{-}, p_{q}^{+}$- граничные значения для $q$-того показателя.

Требования по качеству смеси удобно учесть в виде штрафной составляющей целевой функции:

$$
\begin{aligned}
& f(x)=\sum_{j=1}^{m} c_{j} x_{j}+\mu \sum_{q \in Q} \varphi_{q}^{2}(x) \rightarrow \min , \\
& \left\{\begin{array}{l}
\sum_{j=1}^{m} x_{j}=1, \\
x_{j} \geqslant 0, \quad j=1,2, \ldots, m,
\end{array}\right.
\end{aligned}
$$

где $\mu$ - весовой коэффициент штрафной составляющей; $\varphi_{q}(x)$ - процентное отклонение расчетного значения $q$-того показателя от границы заданного интервала, определяемое по формуле

$$
\varphi_{q}(x)=\left\{\begin{array}{cc}
10^{2}\left(p_{q}^{-}-\phi_{q}(x)\right) / p_{q}^{-}, & p_{q}^{-}-\phi_{q}(x)>0 \\
10^{2}\left(\phi_{q}(x)-p_{q}^{+}\right) / p_{q}^{+}, & p_{q}^{+}-\phi_{q}(x)<0 \\
0, & \phi_{q}(x) \in\left[p_{q}^{-}, p_{q}^{+}\right] .
\end{array}\right.
$$

Для примера решена задача составления трехкомпонентной смеси со следующими условиями. Стоимость $C=1.5 x_{1}+1.3 x_{2}+1.0 x_{3}$. Требования к показателям (октановому числу, плотности, содержанию серы):

$$
\begin{aligned}
& \phi_{1}(x)=90.2 x_{1}+88.5 x_{2}+73.6 x_{3}-14.3 x_{1} x_{2}-9.8 x_{1} x_{3}+28.4 x_{1} x_{2} x_{3} \geqslant 85 \\
& \phi_{2}(x)=0.82 x_{1}+0.59 x_{2}+0.67 x_{3} \leqslant 0.68 \\
& \phi_{3}(x)=0.001 x_{1}+0.003 x_{2}+0.002 x_{3} \leqslant 0.002 .
\end{aligned}
$$

При составлении функции $f(x)$ в $(5)$ используется весовой коэффициент $\mu=10$. По результатам предварительного статистического исследования области изменения $f(x)$ сформировано 10 уровней с шагом $\Delta \zeta=-70$, начиная c $\zeta_{1}=700$.

Для каждого из них выполнено 1000 статистических испытаний. Получены последовательности (3), (4) и построены тренды (см. рисунок):

a) $\psi(l)=0.6916-0.02906 l-0.00374 l^{2}$,

b) $\tilde{x}_{1}(l)=0.33041-0.00145 l+0.000098 l^{2}$,

c) $\tilde{x}_{2}(l)=0.327667+0.013837 l-0.00072 l^{2}$,

d) $\tilde{x}_{3}(l)=0.341923-0.012392 l+0.000626 l^{2}$.

Из уравнения

$$
\psi(l)=a_{0}+a_{1} l+a_{2} l^{2}=0
$$

получено $l^{*}=10.2554$. Подстановкой $l^{*}$ в тренды $\tilde{x}_{1}(l), \tilde{x}_{2}(l), \tilde{x}_{3}(l)$ найдено приближенное решение:

$$
x_{1}^{*}=0.3259, \quad x_{2}^{*}=0.3934, \quad x_{3}^{*}=0.2807 .
$$




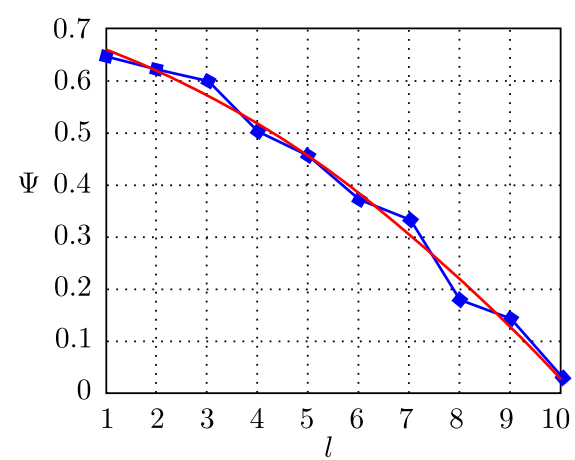

a

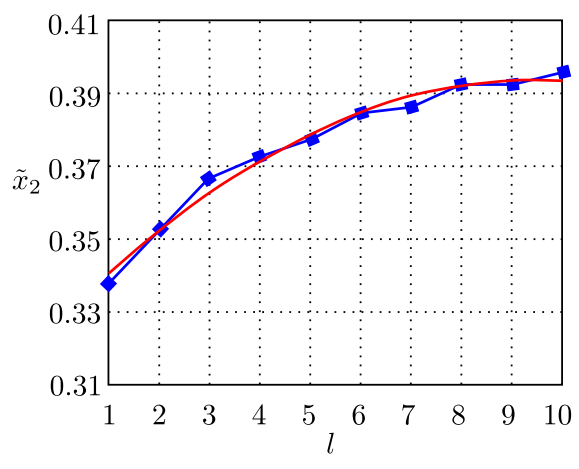

$c$

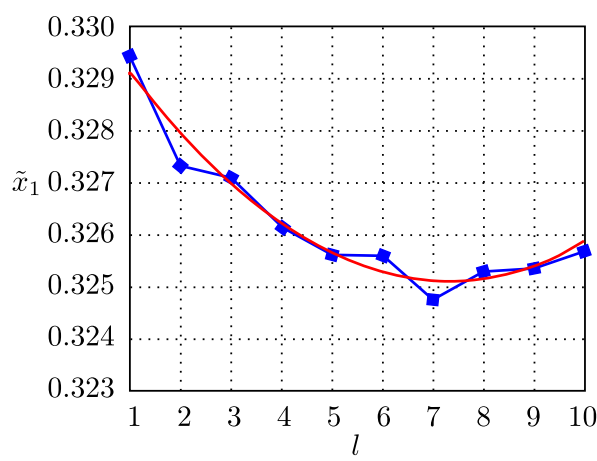

$b$

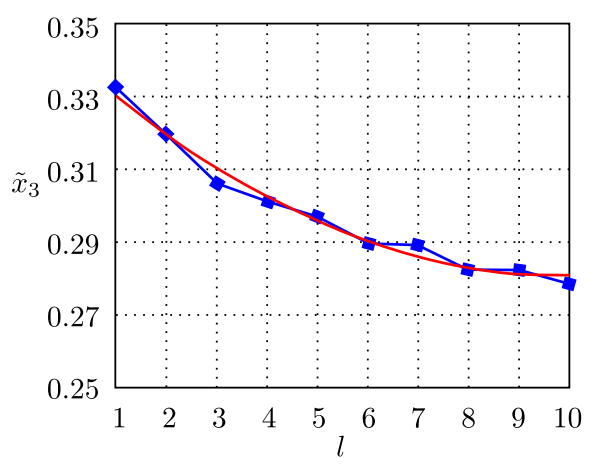

$d$

Тренды результатов статистических испытаний

[Trends of results of statistical tests]

Значение целевой функции (5):

$$
f\left(x^{*}\right)=115.3 .
$$

Уточнение решения осуществляется по описанному выше модифицированному методу отражения правильных симплексов. Проведено 20 серий с уменьшением длины ребра симплекса по формуле

$$
\Delta x_{n}=0.1 n^{-1},
$$

где $n$ - номер серии. Результаты первого приближения $x^{*}$ и уточненного $\breve{x}$ решения представлены в таблице ниже.

\begin{tabular}{c|c|c|c|c|c|c|c}
\hline & $x_{1}$ & $x_{2}$ & $x_{3}$ & $\phi_{1}$ & $\phi_{2}$ & $\phi_{3}$ & $f(x)$ \\
\hline$x^{*}$ & 0.3259 & 0.3934 & 0.2807 & 83.16 & 0.687 & 0.00209 & 115.3 \\
$\breve{x}$ & 0.3702 & 0.3990 & 0.2308 & 83.71 & 0.693 & 0.00206 & 87.6 \\
\hline
\end{tabular}

Как видно из таблицы, уже на первом этапе получено достаточно хорошее приближение. В результате реализации алгоритма выявлена несовместность системы ограничений (6) и получено решение по критерию (5), основная составляющая которого - взвешенная сумма квадратов процентных отклонений показателей от требуемых значений. 
Заключение. В статье предложен метод $\Psi$-преобразования, модифицированный для решения задачи поиска глобального экстремума непрерывной функции на симплексе, имеющей важное прикладное значение. Метод является альтернативой прямому случайному поиску, эффективность которого существенно падает с увеличением числа переменных. Объем вычислений, реализуемых в рамках метода $\Psi$-преобразования, напротив, мало зависит от размерности задачи. Он определяется в основном числом статистических испытаний, которое, по данным [16], не превышает в общей сложности нескольких тысяч и не требует больших затрат машинного времени.

Декларация о финансовых и других взаимоотношениях. Исследование не имело спонсорской поддержки. Все авторы принимали участие в разработке концепции статьи и в написании рукописи. Авторы несут полную ответственность за предоставление окончательной рукописи в печать. Окончательная версия рукописи была одобрена всеми авторами. Авторы не получали гонорар за статью.

\section{ORCIDs}

Михаил Юрьевич Лившиц: http://orcid.org/0000-0001-8027-2273

Александр Павлович Сизиков: http://orcid.org/0000-0001-5067-0676

\section{БИБЛИОГРАФИЧЕСКИЙ СПИСОК}

1. Zhigljavsky A., Žilinskas A. Stochastic Global Optimization/ Springer Optimization and Its Applications. vol. 9. Berlin: Springer, 2008. xiii+262 pp. doi : 10.1007/978-0-387-74740-8.

2. Marti K. Stochastic Optimization Methods/ Applications in Engineering and Operations Research. Berlin: Springer, 2015. xxiv+368 pp. doi: 10.1007/978-3-662-46214-0.

3. Пантелеев А. В. Метаэвристические алгоритмы поиска глобального экстремума. М.: МАИ Принт, 2009. 159 с.

4. M. Tim Jones AI Application Programming/ Programming Series. Boston: Charles River Media, 2003. 496 pp.

5. Савин А. Н., Тимофеева Н. Е. Применение алгоритма оптимизации методом имитации отжига на системах параллельных и распределённых вычислений // Изв. Cарат. унта. Нов. сер. Сер. Математика. Механика. Информатика, 2012. Т. 12, №1. С. 110-116.

6. Botev Z. I., Kroese D. P. Global likelihood optimization via the cross-entropy method, with an application to mixture models / Proceedings of the 2004 Winter Simulation Conference. Washington: IEEE, 2004. pp. 529-535. doi: 10.1109/wsc.2004.1371358.

7. Ernst D., Glavic M., Stan G.-B., Mannor S., Wehenkel L. The cross-entropy method for power system combinatorial optimization problems / 2007 IEEE Lausanne Power Tech. Washington: IEEE, 2007. pp. 1290-1295. doi : 10.1109/pct.2007.4538502.

8. Evans G. E., Keith J. M., Kroese D. P. Parallel cross-entropy optimization / 2007 Winter Simulation Conference. Washington: IEEE, 2007. pp. 2196-2202. doi: 10.1109/wsc. 2007. 4419854.

9. Zhigljavsky A. Theory of Global Random Search / Mathematics and Its Applications (Soviet Series). vol. 65. Berlin: Springer, 1991. xviii+341 pp. doi: 10.1007/978-94-011-3436-1.

10. Феоктистов А. Г., Горский С. А. Реализация метода мультистарта в пакете Градиент // Вестник НГУ Серия: Информационные технологии, 2007. Т. 5, № 2. С. 78-82.

11. Cohoon J., Karro J., Lienig J. Evolutionary Algorithms for the Physical Design of VLSI Circuits / Advances in Evolutionary Computing/ Natural Computing Series; eds. A. Ghosh, S. Tsutsui. Berlin: Springer, 2003. pp. 683-712. doi : 10.1007/978-3-642-18965-4_27.

12. Гладков Л. А., Курейчик В. В., Курейчик В. М. Генетические алгоритмы. М.: Физматлит, 2006. 320 с.

13. Гладков Л. А., Гладкова Н. В. Особенности использования нечетких генетических алгоритмов для решения задач оптимизации и управления // Известия ЮФУ. Технические науки, 2009. № 4(93). С. 130-136. 
14. Skiena S. S. The Algorithm Design Manual. London: Springer, 2008. xvi+730 pp. doi : 10 . 1007/978-1-84800-070-4.

15. Chichinadze V. K. Solution of nonlinear nonconvex optimization problems by $\Psi$-transformation method// Computers \& Mathematics with Applications, 1991. vol.21, no.6-7. pp. $7-15$.

16. Чичинадзе В. К. Решение невыпуклых нелинейных задач оптимизации. Метод Чпреобразования. М.: Наука, 1983. 256 с.

17. Ахмадиев Ф. Г., Гильфанов Р. М. Математическое моделирование и оптимизация "состав-свойство" многокомпонентных смесей// Известия КГАСУ, 2012. №2(20). С. 289-297.

18. Новоселов А. А. Равномерное распределение на стандартном симплексе в $R^{n}$, http: //risktheory.novosyolov.com/lectures/unifs.pdf (дата обращения: 23.10.2016).

19. Русин Ю. В. Алгоритмы статистического моделирования вероятностных распределений. Ярославль: ЯрГУ, 2006. 58 с.

20. Зедгинидзе И. Г. Планирование эксперимента для исследования многокомпонентных систем. М.: Наука, 1976. 390 с.

21. Чинакал В. О. Оптимизация рецептуры светлых нефтепродуктов / Оптимизация, исследование операчий, бионика. М.: Наука, 1973. С. 198-205.

22. Никитин В. А., Мусаев А. А. Оптимизация компаундирования углеводородных смесей // Тр. СПИИРАН, 2007. Т. 4. С. 327-336.

Поступила в редакцию 12/VII/2016;

в окончательном варианте $-23 / \mathrm{X} / 2016$;

принята в печать - 09/XII/2016. 
Vestn. Samar. Gos. Techn. Un-ta. Ser. Fiz.-mat. nauki

[J. Samara State Tech. Univ., Ser. Phys. \& Math. Sci.], 2016, vol. 20, no. 4, pp. 755-768

ISSN: 2310-7081 (online), 1991-8615 (print)

doi: http://dx.doi.org/10.14498/vsgtu1500

MSC: 90C26, 90-08

\title{
METHOD OF SEARCHING FOR GLOBAL EXTREMUM OF A CONTINUOUS FUNCTION ON A SIMPLEX
}

\author{
M. Yu. Livshits, A. P. Sizikov
}

Samara State Technical University,

244, Molodogvardeyskaya st., Samara, 443100, Russian Federation.

\begin{abstract}
A non-convex problem of mathematical programming is considered, which permissible region is a simplex. A two-stage algorithm is proposed for approximate solution of the problem. The region of global optimum is determined using the $\Psi$-transform method at the first stage; local "fine-tuning" of the solution is performed at the second stage. The $\Psi$-transform was modified taking into account the special features of the problem under consideration. $\Psi$-function is determined according to the results of statistical tests implemented using the generator of random points uniformly distributed over the simplex. The proposed method of reflection of regular simplexes is used for fine-tuning of the solution. An example of application of the developed algorithm for solving the problem of optimization of component composition of the hydrocarbon mixture is presented.
\end{abstract}

Keywords: optimization, non-convex problems, $\Psi$ - transform method, uniform distribution over the simplex, multi-component mixtures.

Declaration of Financial and Other Relationships. The research has not had any sponsorship. Each author has participated in the article concept development and in the manuscript writing. The authors are absolutely responsible for submitting the final manuscript in print. Each author has approved the final version of manuscript. The authors have not received any fee for the article.

\section{ORCIDs}

Mikhail Yu. Livshits: http://orcid.org/0000-0001-8027-2273

Aleksandr P. Sizikov: http://orcid.org/0000-0001-5067-0676

(C) 2016 Samara State Technical University.

Please cite this article in press as:

Livshits M. Yu., Sizikov A. P. Method of searching for global extremum of a continuous function on a simplex, Vestn. Samar. Gos. Tekhn. Univ., Ser. Fiz.-Mat. Nauki [J. Samara State Tech. Univ., Ser. Phys. \& Math. Sci.], 2016, vol. 20, no. 4, pp. 755-768. doi: 10.14498/vsgtu1500. (In Russian)

Authors Details:

Mikhail Yu. Livshits (Dr. Techn. Sci.; mikhaillivshits@gmail.com), Head of Dept., Dept. of Management and System Analysis of Thermal Power and Socio-Technical Systems.

Aleksandr P. Sizikov (Cand. Econ. Sci.; apsizikov@mail.ru; Corresponding Author), Doctoral Student, Dept. of Management and System Analysis of Thermal Power and Socio-Technical Systems. 


\section{REFERENCES}

1. Zhigljavsky A., Žilinskas A. Stochastic Global Optimization, Springer Optimization and Its Applications, vol. 9. Berlin, Springer, 2008. xiii+262 pp. doi : 10.1007/978-0-387-74740-8.

2. Marti K. Stochastic Optimization Methods, Applications in Engineering and Operations Research. Berlin, Springer, 2015. xxiv+368 pp. doi: 10.1007/978-3-662-46214-0.

3. Panteleev A. V. Metaevristicheskie algoritmy poiska global'nogo ekstremuma [Metaheuristic algorithms for global extremum search]. Moscow, MAI Print, 2009, 159 pp. (In Russian)

4. M. Tim Jones AI Application Programming, Programming Series. Boston, Charles River Media, 2003, 496 pp.

5. Savin A. N., Timofeeva N. E. The application of optimization algorithm using simulated annealing method for parallel computing systems, Izv. Saratov Univ. (N.S.), Ser. Math. Mech. Inform., 2012, vol. 12, no. 1, pp. 110-116 (In Russian).

6. Botev Z. I., Kroese D. P. Global likelihood optimization via the cross-entropy method, with an application to mixture models, In: Proceedings of the 2004 Winter Simulation Conference. Washington, IEEE, 2004, pp. 529-535. doi : 10.1109/wsc.2004.1371358.

7. Ernst D., Glavic M., Stan G.-B., Mannor S., Wehenkel L. The cross-entropy method for power system combinatorial optimization problems, In: 2007 IEEE Lausanne Power Tech. Washington, IEEE, 2007, pp. 1290-1295. doi: 10.1109/pct.2007.4538502.

8. Evans G. E., Keith J. M., Kroese D. P. Parallel cross-entropy optimization, In: 2007 Winter Simulation Conference. Washington, IEEE, 2007, pp. 2196-2202. doi : 10.1109/wsc. 2007. 4419854.

9. Zhigljavsky A. Theory of Global Random Search, Mathematics and Its Applications (Soviet Series), vol. 65. Berlin, Springer, 1991. xviii+341 pp. doi: 10.1007/978-94-011-3436-1.

10. Feoktistov A. G., Gorskii S. A. The Implementation of the Multi-Start method in the Gradient package, Vestnik NGU Seriia: Informatsionnye tekhnologii, 2007, vol. 5, no. 2, pp. 78-82 (In Russian).

11. Cohoon J., Karro J., Lienig J. Evolutionary Algorithms for the Physical Design of VLSI Circuits, In: Advances in Evolutionary Computing, Natural Computing Series; eds. A. Ghosh, S. Tsutsui. Berlin, Springer, 2003, pp. 683-712. doi : 10.1007/978-3-642-18965-4_27.

12. Gladkov L. A., Kureichik V. V., Kureichik V. M. Geneticheskie algoritmy [Genetic Algorithms]. Moscow, Fizmatlit, 2006, 320 pp. (In Russian)

13. Gladkov L. A., Gladkova N. V. Features of use of fuzzy genetic algorithms for the decision of problems of optimisation and control, Izvestiia YuFU. Tekhnicheskie nauki, 2009, no. 4(93), pp. $130-136$.

14. Skiena S. S. The Algorithm Design Manual. London, Springer, 2008. xvi+730 pp. doi : 10 . 1007/978-1-84800-070-4.

15. Chichinadze V. K. Solution of nonlinear nonconvex optimization problems by $\Psi$-transformation method, Computers \& ${ }^{3}$ Mathematics with Applications, 1991, vol.21, no.6-7, pp. $7-15$.

16. Chichinadze V. K. Reshenie nevypuklykh nelineinykh zadach optimizatsii. Metod $\Psi$ preobrazovaniia [Solution of Nonconvex Nonlinear Optimization Problems. The $\Psi$-transformation method]. Moscow, Nauka, 1983, 256 pp. (In Russian)

17. Akhmadiev F. G., Gil'fanov R. M. Mathematical modeling and optimization of the "compozition-property" of multicomponent mixtures, Izvestiia KGASU, 2012, no. 2(20), pp. 289-297 (In Russian).

18. Novoselov A. A. Uniform distribution on the standard simplex in $R^{n}$ (In Russian), Retrieved http://risktheory.novosyolov.com/lectures/unifs.pdf (October 23, 2016).

19. Rusin Yu. V. Algoritmy statisticheskogo modelirovaniia veroiatnostnykh raspredelenii [Algorithms of statistical modeling of probability distributions]. Yaroslavl, Yaroslavl State Univ., 2006, 58 pp. (In Russian)

20. Zedginidze I. G. Planirovanie eksperimenta dlia issledovaniia mnogokomponentnykh sistem [Experiment Planning for Multicomponent System Study]. Moscow, Nauka, 1976, 390 pp. (In Russian) 
21. Chinakal V. O. Optimization of light oil formulation, In: Optimizatsiia, issledovanie operatsii, bionika [Optimization, Operations Research, Bionics]. Moscow, Nauka, 1973, pp. 198205 (In Russian).

22. Nikitin V. A., Musayev A. A. Optimizing the compounding process of hydrocarbon mixtures, Tr. SPIIRAN, 2007, vol. 4, pp. 327-336 (In Russian).

Received 12/VII/2016;

received in revised form $23 / \mathrm{X} / 2016$;

accepted 09/XII/2016. 